\title{
Some Remarks on Fields of 2-Planes on Compact Smooth 4-Manifolds
}

\author{
Yasuo Matsushita
}

\section{$\S 1 . \quad$ Introduction}

Throughout this paper by a manifold we mean a compact oriented smooth manifold, and by a field of $q$-planes on a manifold a nonsingular field of oriented tangent $q$-planes on it.

It is interesting to observe, as a specific feature in four dimension, that same are the conditions for a 4-manifold to admit the following three different structures:

(A) a field of 2-planes

(B) a pseudo-riemannian metric of signature $(++--)$ with the structure group $S O_{o}(2,2)$

(C) a pair of an almost complex structure and an opposite almost complex structure.

On the basis of such an observation, the purpose of this paper is to discuss some particular aspects of geometry of 4-manifolds which admit fields of 2-planes.

The paper contains two main results: Theorems 4-1 and 7-2, and is organized as follows. $\S 2$ is a quick survay on the problem of fields of 2 -planes on 4-manifolds. In $\S 3$, we consider the reduction of the structure group of the tangent bundle of a 4-manifold which admits a field of 2-planes in connection with twistor spaces. One part of a couple of the main results is given in $\S 4$, which is concerned with the existence of a riemannian metric invariant both by two kinds of almost complex structures on a 4-manifold with a field of 2 -planes. In $\S 5$, we review the irreducible decomposition of the curvature tensors on an almost Hermitian 4-manifold. In $\S 6$, we give an analogue of the irreducible decomposition of the curvature tensors for an opposite almost Hermitian 4-manifold. In the last section $(\S 7)$, the other part of our main results is stated, which is concerned with the irreducible decomposition of the curvature

Received March 6, 1991.

Revised May 28, 1991. 
tensors for an almost Hermitian 4-manifold with two kinds of almost complex structures. We shall see there that such a curvature tensor can be decomposed into eleven irreducible factors under the action of the structure group $G=S O(2) \times S O(2) \cong U(1) \times U(1)$.

\section{$\S 2 . \quad$ General aspects}

We shall give in this section a brief survay of the problem of fields of 2-planes on 4-manifolds. The detailed arguments and results here should be referred to the author's papers [M1], [M2].

It follows from Steenrod's theorem [S, 40.11] that an $n$-manifold admits a pseudo-riemannian metric of signature $(n-q, q)$ with the structure group $S O_{o}(n-q, q)$ if and only if the manifold carries a field of $q$-planes (i.e., the structure group $G$ is reduced to $S O(n-q) \times S O(q))$. The correspondence between (A) and (B) is therefore a special case in dimension $n=4$ with $q=2$, i.e., $G$ is reduced to $S O(2) \times S O(2)$. However, the correspondence between (A) and (C) (or (B) and (C)) is a particular feature in four dimension.

Let $M$ be a 4 -manifold, and $\mu_{M}$ be the intersection form on $H^{2}(M, \mathbb{Z}) /$ Tor. The condition for $M$ to admit a field of 2-planes has been established by Hirzebruch and Hopf [HH, 4.5] as follows: $M$ admits a field of 2-planes if and only if the Hirzebruch index $\tau[M]$ (or the signature) of $M$ and the Euler characteristic $\chi[M]$ of $M$ satisfy a pair of conditions

$$
\begin{aligned}
& 3 \tau[M]+2 \chi[M] \in \Omega(M) \\
& 3 \tau[M]-2 \chi[M] \in \Omega(M),
\end{aligned}
$$

where

$$
\begin{array}{r}
\Omega(M)=\left\{\mu_{M}(w, w) \in \mathbb{Z} \mid w\right. \text { are arbitrary characteristic } \\
\text { elements in } \left.H^{2}(M, \mathbb{Z}) / \text { Tor }\right\} .
\end{array}
$$

As Hirzebruch and Hopf also pointed out [HH, 4.6], the first condition (2-1) is equivalent to the condition (due to $\mathrm{Wu}[\mathrm{W}, \mathrm{p} .74]$ ) for $M$ to admit an almost complex structure. The second condition (2-2) has a similar meaning.

Proposition 2-1. A 4-manifold $M$ admits an almost complex structure whose preferred orientation is opposite to the orientation of $M$ if and only if $M$ satisfies the condition (2-2).

Let $-M$ be $M$ with the orientation reversed. We know that $\tau[-M]=$ $-\tau[M], \chi[-M]=\chi[M]$, and $\Omega(-M)=-\Omega(M)$ (since $\mu_{-M}=-\mu_{M}$ ). 
Thus the condition (2-2) is written in the form

$$
3 \tau[-M]+2 \chi[-M] \in \Omega(-M),
$$

which is just the first condition (2-1) for $-M$. This implies that $-M$ admits an almost complex structure if and only if (2-2) holds.

Definition 2-2. We call an almost complex structure on $-M$ an opposite almost complex structure on $M$.

We thus have a theorem concerning the correspondence between (A) and $(\mathrm{C})$ as follows.

Theorem 2-3. A 4-manifold $M$ admits a field of 2-planes if and only if it admits a pair of an almost complex structure and an opposite almost complex structure.

It is known (Atiyah [A, Theorem 3.1]) that if a $4 k$-dimensional manifold admits a field of $q$-planes $(q \equiv 2 \bmod 4)$, then its Euler characteristic must be even and is congruent to the Hirzebruch index modulo 4. Thus the problem of fields of 2-planes on 4-manifolds is the lowest dimensional examples of Atiyah's theorem. A 4-manifold $M$ with a field of 2-planes must therefore satisfy

$$
\chi[M] \equiv 0 \quad \bmod 2, \quad \chi[M] \equiv \tau[M] \quad \bmod \quad 4,
$$

which will be referred to as Atiyah's condition. In the author's earlier paper [M1], it is shown on the basis of the work of Hirzebruch and Hopf that Aitiyah's condition is sufficient for a simply-connected 4-manifold to admit a field of 2-planes, and hence also the existence condition of (opposite) almost complex structures on such a 4-manifold is established. Recently, Saeki (see [M2, Theorem 2]) obtained a refined and full general version of the theorem of Hirzebruch and Hopf. In fact, he showed that if a 4-manifold has an indefinite intersection form, then Atiyah's condition is also sufficient for the 4-manifold to admit a field of 2-planes, and moreover that if a 4-manifold $M$ has a definite intersection form, then it admits a field of 2-planes if and only if $M$ satisfies Atiyah's condition and

$$
\chi[M]+|\tau[M]| \geq 0 .
$$

It should be noted that he also established the condition for an arbitrary 4-manifold to admit (opposite) almost complex structures [M2, Theorems 8 and 10]. 
We now give a brief discussion on Chern classes. Suppose now that a 4-manifold $M$ admits a field of 2-planes. Then $M$ also admits a pair of an almost complex structure, denoted by $J$, and an opposite almost complex structure, denoted by $J^{\prime}$. The first Chern class $c_{1}(J) \in H^{2}(M, \mathbb{Z})$ determined by $J$ satisfies two conditions ([W], [BPV, Proposition 7.3], $[\mathrm{DK}, 1.1 .7])$ :

$$
\begin{aligned}
c_{1}(J) & \equiv w_{2}(M) \quad \bmod 2 \\
c_{1}^{2}(J)[M] & =3 \tau[M]+2 \chi[M],
\end{aligned}
$$

which correspond to (2-1). Similarly, the opposite almost complex structure $J^{\prime}$ defines a first Chern class $c_{1}\left(J^{\prime}\right)$ which satisfies the conditions

$$
\begin{aligned}
c_{1}\left(J^{\prime}\right) & \equiv w_{2}(M) \quad \bmod 2 \\
c_{1}^{2}\left(J^{\prime}\right)[-M] & =-3 \tau[M]+2 \chi[M],
\end{aligned}
$$

which correspond to (2-2).

The Chern numbers $c_{2}(J)[M]$ and $c_{2}\left(J^{\prime}\right)[-M]$ coincides with each other and also with the Euler characteristic of the manifold:

$$
c_{2}(J)[M]=c_{2}\left(J^{\prime}\right)[-M]=\chi[M] .
$$

It is worthwhile to note some relations

$$
\begin{aligned}
& c_{1}^{2}\left(J^{\prime}\right)[-M]=4 c_{2}(J)[M]-c_{1}^{2}(J)[M] \\
& c_{1}^{2}\left(J^{\prime}\right)[-M]+c_{2}\left(J^{\prime}\right)[-M] \equiv 0 \quad \bmod 12 .
\end{aligned}
$$

The second formula is an analogue of a fundamental relation $c_{1}^{2}(J)[M]+c_{2}(J)[M] \equiv 0 \bmod 12$.

There are many examples of 4-manifolds which admit fields of 2planes. We now restrict, however, our attention to the underlying real 4-manifolds of compact complex surfaces. For such a 4-manifold, it turns out from the above arguments that the pair of conditions (2-1) and (2-2) can be stated in terms of its Chern numbers. In fact, the underlying real 4-manifold of a surface admits a field of 2-planes if and only if $c_{1}^{2}(J)[M]$ $-5 c_{2}(J)[M] \equiv 0 \bmod 12(\Leftrightarrow(2-7 \mathrm{~b}) \Leftrightarrow(2-9 \mathrm{~b}))$

Therefore, the underlying real 4-manifolds of the following (minimal) surfaces [BPV, VI Table 10] admit fields of 2-planes: minimal rational surfaces with $\left(c_{1}^{2}, c_{2}\right)=(8,4)$, Hopf surfaces and Inoue surfaces in the Class 2) of minimal surfaces of class VII, ruled surfaces of genus $g \geq 1$, Enriques surfaces, hyperelliptic surfaces, Kodaira surfaces, K3 surfaces, tori, minimal properly elliptic surfaces, and the surfaces of general type which satisfy (2-7b). 


\section{$\S 3 . \quad$ Grassmann bundles and twistor spaces}

Let $M$ be a 4-manifold. We assume that the structure group $G$ of the tangent bundle of $M$ is already reduced to $S O(4)$, or equivalently there exits a riemmanian metric on $M$. Let $S^{3}$ (the 3-sphere) denote $S U(2)$, the 2-dimensional special unitary group. Then we have an exact sequence

$$
1 \longrightarrow \mathbb{Z}_{2} \longrightarrow S^{3} \times S^{3} \stackrel{\alpha}{\longrightarrow} S O(4) \longrightarrow 1,
$$

where $\alpha$ is a covering map. The product $S^{3} \times S^{3}$ is the spin group of $S O(4)$. If we denote by $S^{1}$ a maximal torus of $S^{3}$, then $\alpha\left(S^{1} \times S^{3}\right)$ is the 2-dimensional unitary group $U(2)$. If we take a maximal torus $S^{1}$ in the second component $S^{3}$ of the spin group $S^{3} \times S^{3}$, then the subgroup $\alpha\left(S^{3} \times S^{1}\right)$ is also a 2-dimensional unitary group, and in this case, is denoted by $U^{\prime}(2)$. The quotient space

$$
S O(4) / U(2)=\alpha\left(S^{3} \times S^{3}\right) / \alpha\left(S^{1} \times S^{3}\right) \cong S^{2}
$$

is the space of almost complex structures on $T M_{x}$ at each point $x \in M$, which are orthogonal with respect to the riemannian metric. Similarly the quotient space

$$
S O(4) / U^{\prime}(2)=\alpha\left(S^{3} \times S^{3}\right) / \alpha\left(S^{3} \times S^{1}\right) \cong S^{2}
$$

is the space of opposite almost complex structures on $T M_{x}$ at each point $x \in M$, which are orthogonal with respect to the riemannian metric.

It is known that the space of oriented tangent 2-planes in $T M_{x}$ at each point $x \in M$ is the Grassmann manifold

$$
G_{2}\left(\mathbb{R}^{4}\right) \cong S O(4) / S O(2) \times S O(2) \cong S^{2} \times S^{2} .
$$

In [HH], Hirzebruch and Hopf obeserved that the first component $S^{2}$ of the above product $S^{2} \times S^{2}$ can be identified with the quotient space $S O(4) / U(2)$, and similarly the second component $S^{2}$ corresponds to the quotient space $S(4) / U^{\prime}(2)$. Thus,

$$
G_{2}\left(\mathbb{R}^{4}\right) \cong S O(4) / U(2) \times S O(4) / U^{\prime}(2) .
$$

Therefore, at each point $x \in M$ the oriented tangent 2-planes are in oneto-one correspondence with the pairs $\left(J_{x}, J_{x}^{\prime}\right)$ of almost complex structures and an opposite almost complex structures.

The bundle over $M$ with the space of such almost complex structures as fibre is the $S O(4) / U(2)$-bundle over $M$, and its total space is known 
as the twistor space of $M$, denoted by $Z^{+}(M)$ (see Atiyah, Hitchin and Singer [AHS]). Similarly, the bundle over $M$ with the space of opposite almost complex structures as fibre is the $S O(4) / U^{\prime}(2)$-bundle over $M$, and its total space is denoted by $Z^{-}(M)$. Thus the Grassmann manifold bundle $G_{2}(M)$ over $M$ with $G_{2}\left(\mathbb{R}^{4}\right)$ as fibre can be written as follows:

$$
G_{2}(M)=Z^{+}(M) \times_{M} Z^{-}(M) .
$$

With this identification, we see that the sections of $G_{2}(M)$ (fields of 2-planes) are in one-to-one correspondence with the pairs of sections of $Z^{+}(M)$ and $Z^{-}(M)$. The conditions (2-1) and (2-2) thus can be interpreted as the conditions for the $S O(4) / U(2)$-bundle $Z^{+}(M)$ and $S O(4) / U^{\prime}(2)$-bundle $Z^{-}(M)$ both to admit sections.

If $M$ admits a field of 2-planes, then the the structure group $G$ is reduced from $S O(4)$ to

$$
\begin{gathered}
\alpha\left(S^{1} \times S^{3}\right) \cap \alpha\left(S^{3} \times S^{1}\right)=\alpha\left(S^{1} \times S^{1}\right) \\
\quad=S O(2) \times S O(2) \cong U(1) \times U(1),
\end{gathered}
$$

and moreover the tangent bundle of $M$ admits also a reduction of $G$ to $S O_{o}(2,2)$ whose maximal compact subgroup is $S O(2) \times S O(2)$, or $M$ carries a pseudo-riemannian metric of signature $(++--)$ with the structure group $S O_{o}(2,2)$. Thus we see again the coincidence (A) $\Leftrightarrow$ (B) $\Leftrightarrow(C)$ from a group theoretical point of view.

\section{$\S 4$. Two kinds of almost complex structures}

Let $M$ be a 4-manifold which admits a field of 2-planes. As we have seen, $M$ admits a pair $\left(J, J^{\prime}\right)$ of an almost complex structure and an opposite almost complex structure.

Consider $M$ to be a riemannian 4-manifold $\left(M, g_{o}\right)$, and choose a pair $\left(J, J^{\prime}\right)$ of two kinds of almost complex structures on $M$, where $g_{o}$ and $\left(J, J^{\prime}\right)$ are arbitrarily chosen. Then we have a riemannian 4-manifold $\left(M, g_{o}\right)$, together with a pair $\left(J, J^{\prime}\right)$, where $J, J^{\prime}$ have at this stage no relation to the metric $g_{o}$. It is well-known, however, that for any almost complex structure $J$ on $\left(M, g_{o}\right)$, we can construct a $J$-invariant metric $g_{1}$ on $M$ as follows:

$$
g_{1}(X, Y)=g_{o}(X, Y)+g_{o}(J X, J Y), \quad \text { for } \quad X, Y \in \mathfrak{X}(M),
$$

where $\mathfrak{X}(M)$ is the algebra of $C^{\infty}$ vector fields on $M$. Similary, there is a $J^{\prime}$-invariant metric $g_{2}$ on $M$ defined by

$$
g_{2}(X, Y)=g_{o}(X, Y)+g_{o}\left(J^{\prime} X, J^{\prime} Y\right), \quad \text { for } \quad X, Y \in \mathfrak{X}(M) .
$$


Here by $J$-invariance (resp. $J^{\prime}$-invariance), we mean that $g_{1}(J X, J Y)=$ $g_{1}(X, Y)$ (resp. $\left.g_{2}\left(J^{\prime} X, J^{\prime} Y\right)=g_{2}(X, Y)\right)$. We thus obtain two kinds of almost Hermitian 4-manifolds $\left(M, g_{1}, J\right)$ and $\left(M, g_{2}, J^{\prime}\right)$. Such a metric $g_{1}$ (resp. $g_{2}$ ) is not in general invariant by $J^{\prime}$ (resp. $J$ ).

For such a 4-manifold with two kinds of almost complex structures, we are led to consider a question: Does $M$ admit a riemannian metric which is invariant by both almost complex structures $J$ and $J^{\prime}$ ? We have one of the main results as follows.

Theorem 4-1. Let $M$ be a 4-manifold which admits a field of 2planes. Associated with each field $\tau$ of 2-planes on $M$, there exits on $M$ a pair of an almost complex structure $J_{\tau}$ and an opposite almost complex structure $J_{\tau}^{\prime}$, together with an invariant riemannian metric $g$ such that (i) $J_{\tau}$ and $J_{\tau}^{\prime}$ commute with each other,

(ii) $g$ is invariant by both $J_{\tau}$ and $J_{\tau}^{\prime}$.

We shall prove this theorem by constructing such two kinds of almost complex structures.

Let $\tau$ be a field of 2-planes on $M$, and $g_{o}$ a riemannian metric on $M$, both of which are arbitrarily chosen. Associated with the field $\tau$ of 2 -planes, we can choose a local orthonormal frame $\left\{e_{1}, e_{2}, e_{3}, e_{4}\right\}$ at each point $x$ of $M$ with respect to $g_{o}$ such that

$$
e_{1}, e_{2} \in \tau_{x}, \quad e_{3}, e_{4} \in \nu_{x},
$$

where $\nu$ is the 2-dimensional subbundle of the tangent bundle consisting of normal vectors to $\tau$. Relative to such a frame, we can always construct locally a couple of rank 2 tensor fields $J_{\tau}$ and $J_{\tau}^{\prime}$ of type $(1,1)$ at each point $x \in M$ as follows:

$$
\begin{aligned}
& \left(J_{\tau}\right)_{x}=e^{1} \otimes e_{2}-e^{2} \otimes e_{1}+e^{3} \otimes e_{4}-e^{4} \otimes e_{3}, \\
& \left(J_{\tau}^{\prime}\right)_{x}=e^{1} \otimes e_{2}-e^{2} \otimes e_{1}-e^{3} \otimes e_{4}+e^{4} \otimes e_{3},
\end{aligned}
$$

where $\left\{e^{1}, e^{2}, e^{3}, e^{4}\right\}$ is the local dual orthonormal basis of 1-forms such that $e^{i}\left(e_{j}\right)=\delta_{i j}$. Note that

$$
\begin{aligned}
& \left(J_{\tau}\right)_{x}\left(e_{1}\right)=e_{2}, \quad\left(J_{\tau}\right)_{x}\left(e_{2}\right)=-e_{1}, \quad\left(J_{\tau}\right)_{x}\left(e_{3}\right)=e_{4}, \quad\left(J_{\tau}\right)_{x}\left(e_{4}\right)=-e_{3}, \\
& \left(J_{\tau}^{\prime}-5 \mathrm{~b}\right) \\
& \left(J_{x}^{\prime}\left(e_{1}\right)=e_{2}, \quad\left(J_{\tau}^{\prime}\right)_{x}\left(e_{2}\right)=-e_{1}, \quad\left(J_{\tau}^{\prime}\right)_{x}\left(e_{3}\right)=-e_{4}, \quad\left(J_{\tau}^{\prime}\right)_{x}\left(e_{4}\right)=e_{3} .\right.
\end{aligned}
$$

Lemma 4-2. $\quad J_{\tau}$ and $J_{\tau}^{\prime}$ are globally defined nonsingular tensor fields of type $(1,1)$ on $M$. 
Proof. If we choose another orthonormal frame $\left\{\hat{e}_{1}, \hat{e}_{2}, \hat{e}_{3}, \hat{e}_{4}\right\}$ with the same property (4-3) $\left(\hat{e}_{1}, \hat{e}_{2} \in \tau_{x}, \hat{e}_{3}, \hat{e}_{4} \in \nu_{x}\right)$, then it is locally related to the frame $\left\{e_{1}, e_{2}, e_{3}, e_{4}\right\}$ by

$$
\left[\begin{array}{l}
\hat{e}_{1} \\
\hat{e}_{2} \\
\hat{e}_{3} \\
\hat{e}_{4}
\end{array}\right]=\left[\begin{array}{cccc}
\cos \theta & -\sin \theta & 0 & 0 \\
\sin \theta & \cos \theta & 0 & 0 \\
0 & 0 & \cos \phi & -\sin \phi \\
0 & 0 & \sin \phi & \cos \phi
\end{array}\right]\left[\begin{array}{l}
e_{1} \\
e_{2} \\
e_{3} \\
e_{4}
\end{array}\right]
$$

for some $\theta, \phi \in \mathbb{R}$, since the structure group $G$ of the tangent bundle is reduced to $S O(2) \times S O(2)$.

Associated with the new frame $\left\{\hat{e}_{1}, \hat{e}_{2}, \hat{e}_{3}, \hat{e}_{4}\right\}$, we take the tensors $\left(\hat{J}_{\tau}\right)_{x}$ and $\left(\hat{J}_{\tau}^{\prime}\right)_{x}$ as defined by the similar forms to $\left(J_{\tau}\right)_{x}$ and $\left(J_{\tau}^{\prime}\right)_{x}$ as follows

$$
\begin{aligned}
& \left(\hat{J}_{\tau}\right)_{x}=\hat{e}^{1} \otimes \hat{e}_{2}-\hat{e}^{2} \otimes \hat{e}_{1}+\hat{e}^{3} \otimes \hat{e}_{4}-\hat{e}^{4} \otimes \hat{e}_{3}, \\
& \left(\hat{J}_{\tau}^{\prime}\right)_{x}=\hat{e}^{1} \otimes \hat{e}_{2}-\hat{e}^{2} \otimes \hat{e}_{1}-\hat{e}^{3} \otimes \hat{e}_{4}+\hat{e}^{4} \otimes \hat{e}_{3} .
\end{aligned}
$$

Using the transformation law (4-6) of the frames, we can easily verify the following coincidences in the overlap region where both frames are defined:

$$
\left(\hat{J}_{\tau}\right)_{x}=\left(J_{\tau}\right)_{x}, \quad\left(\hat{J}_{\tau}^{\prime}\right)_{x}=\left(J_{\tau}^{\prime}\right)_{x} .
$$

This implies that $J_{\tau}$ and $J_{\tau}^{\prime}$ are both globally defined nonsingular tensor fields of type $(1,1)$ due to the existence of the field $\tau$ of 2-planes.

Q.E.D.

It is clear that as the endomorphisms of the tangent bundle, $J_{\tau}$ and $J_{\tau}^{\prime}$ have the property

$$
J_{\tau}^{2}=-1, \quad{J^{\prime}}_{\tau}^{2}=-1
$$

Lemma 4-3. $\quad J_{\tau} J_{\tau}^{\prime}=J_{\tau}^{\prime} J_{\tau}$. $5 b)$.

Proof. Such a commutativity is easily verified from (4-5a) and (4Q.E.D.

Proof of Theorem 4-1. In the same spirit of constructing invariant metrics $g_{1}$ and $g_{2}$, we define a new metric $g$ as follows: for $X, Y \in \mathfrak{X}(M)$

$$
\begin{aligned}
g(X, Y)=g_{o}(X, Y) & +g_{o}\left(J_{\tau} X, J_{\tau} Y\right) \\
& +g_{o}\left(J_{\tau}^{\prime} X, J_{\tau}^{\prime} Y\right)+g_{o}\left(J_{\tau} J_{\tau}^{\prime} X, J_{\tau} J_{\tau}^{\prime} Y\right) .
\end{aligned}
$$

It is easy to see that $g$ is invariant by both $J_{\tau}$ and $J_{\tau}^{\prime}$, since $J_{\tau}$ and $J_{\tau}^{\prime}$ cummute with each other. Such a metric $g$ is the desired one. Q.E.D. 
We thus obtain a quadruple $\left(M, g, J_{\tau}, J_{\tau}^{\prime}\right)$, which will be the main concern of the remainder of the paper.

\section{$\S 5$. Almost Hermitian 4-manifolds $\left(M, g, J_{\tau}\right)$}

The contents of this section are all known facts, but they are important and are the prototypes of our results treated in the subsequent two sections.

An almost Hermitian 4-manifold $\left(M, g, J_{\tau}, J_{\tau}^{\prime}\right)$ with two kinds of almost complex structures clearly carries two kinds of Kähler forms $F_{\tau}$ and $F_{\tau}^{\prime}$, defined as follows: for $X, Y \in \mathfrak{X}(M)$

$$
F_{\tau}(X, Y)=g\left(J_{\tau} X, Y\right), \quad F_{\tau}^{\prime}(X, Y)=g\left(J_{\tau}^{\prime} X, Y\right) .
$$

As stated before, the tangent bundle of $M$ admits a reduction of the structure group $G$ to $U(1) \times U(1)$.

If we forget two kinds of almost complex structures $J_{\tau}, J_{\tau}^{\prime}$ from the quadruple $\left(M, g, J_{\tau}, J_{\tau}^{\prime}\right)$, then the bundle of 2 -forms over the riemannian 4-manifold $(M, g)$ with the structure group $G=S O(4)$ splits into two subbundles

$$
\Lambda^{2}(M)=\Lambda_{+} \oplus \Lambda_{-},
$$

where $\Lambda_{ \pm}$are \pm 1 eigenspaces of the Hodge star operator.

Although $M$ admits two kinds of almost complex structures $J_{\tau}$ and $J_{\tau}^{\prime}$, we forget $J_{\tau}^{\prime}$ for a moment and cosider the triple $\left(M, g, J_{\tau}\right)$, an almost Hermitian 4-manifold. The structure group $G$ is the unitary group $U(2)\left(=\alpha\left(S^{1} \times S^{3}\right)\right)$. It is well-known that under the action of $U(2)$, the bundle $\Lambda^{2}(M)$ of 2-forms over the triple $\left(M, g, J_{\tau}\right)$ splits further as follows:

$$
\Lambda^{2}(M)=\mathbb{R} F_{\tau} \oplus L_{\tau} \oplus \Lambda_{-},
$$

where $\mathbb{R} F_{\tau}$ is the 1-dimensional subbundle spanned by the Kähler form $F_{\tau}, L_{\tau}$ the 2-dimensional subbundle of $J_{\tau}$-skew invariant 2 -forms

$$
L_{\tau}=\left\{\Phi \in \Lambda^{2}(M) \mid J_{\tau} \cdot \Phi=-\Phi\right\} .
$$

Moreover, it should be noted that the sum $\mathbb{R} F_{\tau} \oplus \Lambda_{-}$consists of $J_{\tau^{-}}$ invariant 2-forms, and that the sum $\mathbb{R} F_{\tau} \oplus L_{\tau}$ coincides with $\Lambda_{+}$.

Associated with the splitting (5-3), the bundle $\mathcal{R}(M)$ of the curvature tensors over $\left(M, g, J_{\tau}\right)$ splits into a direct sum of seven irreducible factors [TV, Theorem 14.3]:

$$
\begin{aligned}
\mathcal{R}(M)=\mathcal{L}\left(\pi_{1}\right) & \oplus \mathcal{L}\left(\pi_{1}-\pi_{2}\left(J_{\tau}\right)\right) \oplus \mathcal{W}_{7}\left(J_{\tau}\right) \oplus \mathcal{W}_{9}\left(J_{\tau}\right) \\
& \oplus \mathcal{R}_{W}^{-} \oplus \mathcal{W}_{2}\left(J_{\tau}\right) \oplus \mathcal{W}_{8}\left(J_{\tau}\right)
\end{aligned}
$$


where with the aid of notation: $\pi_{2}\left(J_{\tau}\right)=-2 *-3 J_{\tau}+2$, we have put

$$
\begin{aligned}
\mathcal{L}\left(\pi_{1}-\pi_{2}\left(J_{\tau}\right)\right)=\operatorname{span}\left\{2 *+3 J_{\tau}-1\right\} & \\
\mathcal{W}_{7}\left(J_{\tau}\right) & =\{R \in \mathcal{R}(M) \mid R *=R\} \cap \mathcal{L}^{\perp}\left(\pi_{1}-\pi_{2}\left(J_{\tau}\right)\right) \\
\mathcal{W}_{9}\left(J_{\tau}\right) & =\left\{R \in \mathcal{R}(M) \mid R *=R, R J_{\tau}=-J_{\tau} R\right\} \\
\mathcal{R}_{W}^{-} & =\{R \in \mathcal{R}(M) \mid R *=-R\} \\
\mathcal{W}_{2}\left(J_{\tau}\right) & =\left\{R \in \mathcal{R}(M) \mid R *=-* R, R J_{\tau}=R\right\} \\
\mathcal{W}_{8}\left(J_{\tau}\right) & =\left\{R \in \mathcal{R}(M) \mid R *=-* R, \quad R J_{\tau}=-J_{\tau} R\right\} .
\end{aligned}
$$

Further, we have

$$
\begin{gathered}
\operatorname{dim} \mathcal{L}\left(\pi_{1}\right)=\operatorname{dim} \mathcal{L}\left(\pi_{1}-\pi_{2}\left(J_{\tau}\right)\right)=1, \quad \operatorname{dim} \mathcal{W}_{7}\left(J_{\tau}\right)=\mathcal{W}_{9}\left(J_{\tau}\right)=2, \\
\operatorname{dim} \mathcal{R}_{W}^{-}=5, \quad \operatorname{dim} \mathcal{W}_{2}\left(J_{\tau}\right)=3, \quad \operatorname{dim} \mathcal{W}_{8}\left(J_{\tau}\right)=6 .
\end{gathered}
$$

Moreover, the sum $\mathcal{L}\left(\pi_{1}-\pi_{2}\left(J_{\tau}\right)\right) \oplus \mathcal{W}_{7}\left(J_{\tau}\right) \oplus \mathcal{W}_{9}\left(J_{\tau}\right)$ coincides with the bundle $\mathcal{R}_{W}^{+}$of self-dual Weyl curvature tensors, and the sum $\mathcal{W}_{2}\left(J_{\tau}\right)$ $\oplus \mathcal{W}_{8}\left(J_{\tau}\right)$ is the bundle $\mathcal{R}_{o}$ of traceless Ricci tensors. Thus, the existence of $J_{\tau}$ induces the splitting $\mathcal{R}_{W}^{+}$into three factors and $\mathcal{R}_{o}$ into two factors.

\section{$\S 6 . \quad$ Opposite almost Hermitian 4-manifolds $\left(M, g, J_{\tau}^{\prime}\right)$}

In this section, we consider the irreducible decomposition of the curvature tensors of an opposite almost Hermitian 4-manifold $(M, g$, $\left.J_{\tau}^{\prime}\right)$, which is obtained by deleting $J_{\tau}$ from the quadruple $\left(M, g, J_{\tau}, J_{\tau}^{\prime}\right)$.

The structure group $G$ is now the unitary group $U^{\prime}(2)\left(=\alpha\left(S^{3} \times\right.\right.$ $\left.S^{1}\right)$ ). For the bundle of 2 -forms over $\left(M, g, J_{\tau}^{\prime}\right)$, we have a decomposition similar to (5-3) for $\left(M, g, J_{\tau}\right)$.

Proposition 6-1. For an opposite almost Hermitian 4-manifold $\left(M, g, J_{\tau}^{\prime}\right)$, the bundle $\Lambda^{2}(M)$ of 2 -forms over $M$ splits into a direct sum

$$
\Lambda^{2}(M)=\Lambda_{+} \oplus \mathbb{R} F_{\tau}^{\prime} \oplus L_{\tau}^{\prime},
$$

where $\mathbb{R} F_{\tau}^{\prime}$ is the 1-dimensional subbundle spanned by the Kähler form $F_{\tau}^{\prime}, L_{\tau}^{\prime}$ the 2-dimensional subbundle of $J_{\tau}^{\prime}$-skew invariant 2-forms

$$
L_{\tau}^{\prime}=\left\{\Phi \in \Lambda^{2}(M) \mid J_{\tau}^{\prime} \cdot \Phi=-\Phi\right\} .
$$

Moreover, the sum $\mathbb{R} F_{\tau}^{\prime} \oplus \Lambda_{+}$consistes of $J_{\tau}^{\prime}$-invariant 2-forms, and the sum $\mathbb{R} F_{\tau}^{\prime} \oplus L_{\tau}^{\prime}$ coincides with $\Lambda_{-}$.

Associated with the above splitting of $\Lambda^{2}(M)$, we have the following (cf. [TV, Theorem 14.3]). 
Proposition 6-2. For an opposite almost Hermitian 4-manifold $\left(M, g, J_{\tau}^{\prime}\right)$, the bundle $\mathcal{R}(M)$ of the curvature tensors over $M$ splits into a direct sum of seven irreducible factors under the action of the structure group $U^{\prime}(2)$ :

$$
\begin{aligned}
\mathcal{R}(M)=\mathcal{L}\left(\pi_{1}\right) & \oplus \mathcal{L}\left(\pi_{1}-\pi_{2}\left(J_{\tau}^{\prime}\right)\right) \oplus \mathcal{W}_{7}\left(J_{\tau}^{\prime}\right) \oplus \mathcal{W}_{9}\left(J_{\tau}^{\prime}\right) \\
& \oplus \mathcal{R}_{W}^{+} \oplus \mathcal{W}_{2}\left(J_{\tau}^{\prime}\right) \oplus \mathcal{W}_{8}\left(J_{\tau}^{\prime}\right)
\end{aligned}
$$

where

$$
\begin{gathered}
\mathcal{L}\left(\pi_{1}-\pi_{2}\left(J_{\tau}^{\prime}\right)\right)=\operatorname{span}\left\{2 *+3 J_{\tau}^{\prime}-1\right\} \\
\mathcal{W}_{7}\left(J_{\tau}^{\prime}\right)=\{R \in \mathcal{R}(M) \mid R *=-R\} \cap \mathcal{L}^{\perp}\left(\pi_{1}-\pi_{2}\left(J_{\tau}^{\prime}\right)\right) \\
\mathcal{W}_{9}\left(J_{\tau}^{\prime}\right)=\left\{R \in \mathcal{R}(M) \mid R *=-R, R J_{\tau}^{\prime}=-J_{\tau}^{\prime} R\right\} \\
\mathcal{R}_{W}^{+}=\{R \in \mathcal{R}(M) \mid R *=R\} \\
\mathcal{W}_{2}\left(J_{\tau}^{\prime}\right)=\left\{R \in \mathcal{R}(M) \mid R *=-* R, R J_{\tau}^{\prime}=R\right\} \\
\mathcal{W}_{8}\left(J_{\tau}^{\prime}\right)=\left\{R \in \mathcal{R}(M) \mid R *=-* R, R J_{\tau}^{\prime}=-J_{\tau}^{\prime} R\right\} .
\end{gathered}
$$

Further, we have

$$
\begin{gathered}
\operatorname{dim} \mathcal{L}\left(\pi_{1}\right)=\operatorname{dim} \mathcal{L}\left(\pi_{1}-\pi_{2}\left(J_{\tau}^{\prime}\right)\right)=1, \quad \operatorname{dim} \mathcal{W}_{7}\left(J_{\tau}^{\prime}\right)=\mathcal{W}_{9}\left(J_{\tau}^{\prime}\right)=2, \\
\operatorname{dim} \mathcal{R}_{W}^{-}=5, \quad \operatorname{dim} \mathcal{W}_{2}\left(J_{\tau}^{\prime}\right)=3, \quad \operatorname{dim} \mathcal{W}_{8}\left(J_{\tau}^{\prime}\right)=6 .
\end{gathered}
$$

Moreover, the sum $\mathcal{L}\left(\pi_{1}-\pi_{2}\left(J_{\tau}^{\prime}\right)\right) \oplus \mathcal{W}_{7}\left(J_{\tau}^{\prime}\right) \oplus \mathcal{W}_{9}\left(J_{\tau}^{\prime}\right)$ coincides with the bundle $\mathcal{R}_{W}^{-}$of anti-self-dual Weyl curvature tensors, and the sum $\mathcal{W}_{2}\left(J_{\tau}^{\prime}\right) \oplus \mathcal{W}_{8}\left(J_{\tau}^{\prime}\right)$ is the bundle $\mathcal{R}_{o}$ of traceless Ricci tensors.

Thus, the existence of $J_{\tau}^{\prime}$ induces the splitting $\mathcal{R}_{W}^{-}$into three factors and $\mathcal{R}_{o}$ into two factors.

\section{$\S 7$. Almost Hermitian 4-manifolds $\left(M, g, J_{\tau}, J_{\tau}^{\prime}\right)$ with two kinds of almost complex structures}

In this section we shall state the second part of our main results concerning the irreducible decomposition of the curvature tensor on an almost Hermitian 4-manifold $\left(M, g, J_{\tau}, J_{\tau}^{\prime}\right)$ with two kinds of almost complex structures.

Now the structure group $G$ is reduced to $U(1) \times U(1)$. Such a reduction induces a further splitting of the bundle $\Lambda^{2}(M)$.

Proposition 7-1. The bundle $\Lambda^{2}(M)$ of 2-forms over an almost Hermitian 4-manifold $\left(M, g, J_{\tau}, J_{\tau}^{\prime}\right)$ with two kinds of almost complex 
structures splits into a direct sum of four subbundles under the action of $G=U(1) \times U(1)$ as follows

$$
\Lambda^{2}(M)=\mathbb{R} F_{\tau} \oplus L_{\tau} \oplus \mathbb{R} F_{\tau}^{\prime} \oplus L_{\tau}^{\prime},
$$

where $\mathbb{R} F_{\tau}, \mathbb{R} F_{\tau}^{\prime}$ are respectively the 1-dimensional subbundles spanned by the Kähler forms $F_{\tau}, F_{\tau}^{\prime}$, and $L_{\tau}, L_{\tau}^{\prime}$ are the 2-dimensional subbundles of $J_{\tau}, J_{\tau}^{\prime}$ skew invariant 2-forms:

$$
\begin{aligned}
& L_{\tau}=\left\{\Phi \in \Lambda^{2}(M) \mid J_{\tau} \cdot \Phi=-\Phi\right\} \\
& L_{\tau}^{\prime}=\left\{\Phi \in \Lambda^{2}(M) \mid J_{\tau}^{\prime} \cdot \Phi=-\Phi\right\} .
\end{aligned}
$$

Proof. Since $U(1) \times U(1) \subset \alpha\left(S^{1} \times S^{3}\right)$, the action of the structure group induces a splitting: $\Lambda_{+}^{2}=\mathbb{R} F_{\tau} \oplus L_{\tau}$. Similarly, $U(1) \times U(1) \subset$ $\alpha\left(S^{3} \times S^{1}\right)$, and hence we have $\Lambda_{-}^{2}=\mathbb{R} F_{\tau}^{\prime} \oplus L_{\tau}^{\prime}$.

Q.E.D.

Note that $\mathbb{R} F_{\tau} \oplus \mathbb{R} F_{\tau}^{\prime}=\left\{\Phi \in \Lambda^{2}(M) \mid J_{\tau} \cdot \Phi=\Phi, \quad J_{\tau}^{\prime} \cdot \Phi=\Phi\right\}$.

At this stage, based on the splitting of $\Lambda^{2}(M)$ we can state the second part of our main results on the decomposition of $\mathcal{R}(M)$ into irreducible factors.

Theorem 7-2. For an almost Hermitian 4-manifold $\left(M, g, J_{\tau}\right.$, $\left.J_{\tau}^{\prime}\right)$ with two kinds of almost complex structures, the bundle $\mathcal{R}(M)$ of the curvature tensors over $M$ splits into a direct sum of eleven irreducible factors under the action of $G=U(1) \times U(1)$ :

$$
\begin{gathered}
\mathcal{R}(M)=\mathcal{L}\left(\pi_{1}\right) \oplus \mathcal{L}\left(\pi_{1}-\pi_{2}\left(J_{\tau}\right)\right) \oplus \mathcal{W}_{7}\left(J_{\tau}\right) \oplus \mathcal{W}_{9}\left(J_{\tau}\right) \\
\oplus \mathcal{L}\left(\pi_{1}-\pi_{2}\left(J_{\tau}^{\prime}\right)\right) \oplus \mathcal{W}_{7}\left(J_{\tau}^{\prime}\right) \oplus \mathcal{W}_{9}\left(J_{\tau}^{\prime}\right) \\
\oplus \mathcal{W}_{A} \oplus \mathcal{W}_{B} \oplus \mathcal{W}_{C} \oplus \mathcal{W}_{D}
\end{gathered}
$$

where

$$
\begin{array}{ll}
\mathcal{W}_{A}=\{R \in \mathcal{R}(M) \mid R *=-* R, & \left.R J_{\tau}=R, R J_{\tau}^{\prime}=R\right\} \\
\mathcal{W}_{B}=\{R \in \mathcal{R}(M) \mid R *=-* R, & \left.R J_{\tau}=R, R J_{\tau}^{\prime}=-J_{\tau}^{\prime} R\right\} \\
\mathcal{W}_{C}=\{R \in \mathcal{R}(M) \mid R *=-* R, & \left.R J_{\tau}=-J_{\tau} R, R J_{\tau}^{\prime}=R\right\} \\
\mathcal{W}_{D}=\{R \in \mathcal{R}(M) \mid R *=-* R, & \left.R J_{\tau}=-J_{\tau} R, R J_{\tau}^{\prime}=-J_{\tau}^{\prime} R\right\} .
\end{array}
$$

Further, we have

$$
\begin{gathered}
\operatorname{dim} \mathcal{L}\left(\pi_{1}\right)=\operatorname{dim} \mathcal{L}\left(\pi_{1}-\pi_{2}\left(J_{\tau}\right)\right)=\operatorname{dim} \mathcal{L}\left(\pi_{1}-\pi_{2}\left(J_{\tau}^{\prime}\right)\right)=\operatorname{dim} \mathcal{W}_{A}=1 \\
\operatorname{dim} \mathcal{W}_{7}\left(J_{\tau}\right)=\operatorname{dim} \mathcal{W}_{7}\left(J_{\tau}^{\prime}\right)=\operatorname{dim} \mathcal{W}_{9}\left(J_{\tau}\right)=\operatorname{dim} \mathcal{W}_{9}\left(J_{\tau}^{\prime}\right) \\
=\operatorname{dim} \mathcal{W}_{B}=\operatorname{dim} \mathcal{W}_{C}=2, \quad \operatorname{dim} \mathcal{W}_{D}=4
\end{gathered}
$$


Proof. We may assume that the bundle $\mathcal{R}(M)$ has been decomposed as (5-5) for the triple $\left(M, g, J_{\tau}\right)$. We shall show, due to the existence $J_{\tau}^{\prime}$, the following three: (i) the bundle $\mathcal{R}_{W}^{-}$of anti-self-dual Weyl curvatures splits into a sum of three irreducible factors:

$$
\mathcal{R}_{W}^{-}(M)=\mathcal{L}\left(\pi_{1}-\pi_{2}\left(J_{\tau}^{\prime}\right)\right) \oplus \mathcal{W}_{7}\left(J_{\tau}^{\prime}\right) \oplus \mathcal{W}_{9}\left(J_{\tau}^{\prime}\right),
$$

(ii) $\mathcal{W}_{2}\left(J_{\tau}\right)$ into two irreducible factors:

$$
\mathcal{W}_{2}\left(J_{\tau}\right)=\mathcal{W}_{A} \oplus \mathcal{W}_{B}
$$

and (iii) $\mathcal{W}_{8}\left(J_{\tau}\right)$ into two irreducible factors:

$$
\mathcal{W}_{8}\left(J_{\tau}\right)=\mathcal{W}_{C} \oplus \mathcal{W}_{D}
$$

(Note that $\mathcal{W}_{2}\left(J_{\tau}^{\prime}\right)=\mathcal{W}_{A} \oplus \mathcal{W}_{C}$, and $\mathcal{W}_{8}\left(J_{\tau}^{\prime}\right)=\mathcal{W}_{B} \oplus \mathcal{W}_{D}$ )

Concerning (i), we must recognize first that $\mathcal{R}_{W}^{-}(M) \subset \operatorname{End}\left(\Lambda_{-}\right)$. From Proposition 6-1, $\operatorname{End}\left(\Lambda_{-}\right)$splits as follows:

$$
\begin{aligned}
\operatorname{End}\left(\Lambda_{-}\right) & =\operatorname{End}\left(\mathbb{R} F_{\tau}^{\prime} \oplus L_{\tau}^{\prime}\right) \\
= & \operatorname{End}\left(\mathbb{R} F_{\tau}^{\prime}\right) \oplus \operatorname{Hom}\left(\mathbb{R} F_{\tau}^{\prime}, L_{\tau}^{\prime}\right) \oplus \operatorname{Hom}\left(L_{\tau}^{\prime}, \mathbb{R} F_{\tau}^{\prime}\right) \oplus \operatorname{End}\left(L_{\tau}^{\prime}\right) .
\end{aligned}
$$

Thus, it is easy to see that $\mathcal{R}_{W}^{-}(M)$ consists of three factors:

$$
\begin{aligned}
\mathcal{L}\left(\pi_{1}-\pi_{2}\left(J_{\tau}^{\prime}\right)\right) & \subset \operatorname{End}\left(\mathbb{R} F_{\tau}^{\prime}\right) \\
\mathcal{W}_{7}\left(J_{\tau}^{\prime}\right) & \subset \operatorname{Hom}\left(\mathbb{R} F_{\tau}^{\prime}, L_{\tau}^{\prime}\right) \oplus \operatorname{Hom}\left(L_{\tau}^{\prime}, \mathbb{R} F_{\tau}^{\prime}\right) \\
\mathcal{W}_{9}\left(J_{\tau}^{\prime}\right) & \subset \operatorname{End}\left(L_{\tau}^{\prime}\right) .
\end{aligned}
$$

For (ii), we know that $\mathcal{W}_{2}\left(J_{\tau}\right) \subset \operatorname{Hom}\left(\mathbb{R} F_{\tau}, \Lambda_{-}\right) \oplus \operatorname{Hom}\left(\Lambda_{-}, \mathbb{R} F_{\tau}\right)$. Since $\Lambda_{-}=\mathbb{R} F_{\tau}^{\prime} \oplus L_{\tau}^{\prime}$, we see that $\mathcal{W}_{2}\left(J_{\tau}\right)$ splits into two factors

$$
\begin{aligned}
& \mathcal{W}_{A} \subset \operatorname{Hom}\left(\mathbb{R} F_{\tau}, \mathbb{R} F_{\tau}^{\prime}\right) \oplus \operatorname{Hom}\left(\mathbb{R} F_{\tau}^{\prime}, \mathbb{R} F_{\tau}\right) \\
& \mathcal{W}_{B} \subset \operatorname{Hom}\left(\mathbb{R} F_{\tau}, L_{\tau}^{\prime}\right) \oplus \operatorname{Hom}\left(L_{\tau}^{\prime}, \mathbb{R} F_{\tau}\right) .
\end{aligned}
$$

For the last case (iii), we know that $\mathcal{W}_{8}\left(J_{\tau}\right) \subset \operatorname{Hom}\left(L_{\tau}, \Lambda_{-}\right) \oplus$ $\operatorname{Hom}\left(\Lambda_{-}, L_{\tau}\right)$. Due to the splitting $\Lambda_{-}=\mathbb{R} F_{\tau}^{\prime} \oplus L_{\tau}^{\prime}$, we see that $\mathcal{W}_{8}\left(J_{\tau}\right)$ splits into two factors:

$$
\begin{aligned}
& \mathcal{W}_{C} \subset \operatorname{Hom}\left(L_{\tau}, \mathbb{R} F_{\tau}^{\prime}\right) \oplus \operatorname{Hom}\left(\mathbb{R} F_{\tau}^{\prime}, L_{\tau}\right) \\
& \mathcal{W}_{D} \subset \operatorname{Hom}\left(L_{\tau}, L_{\tau}^{\prime}\right) \oplus \operatorname{Hom}\left(L_{\tau}^{\prime}, L_{\tau}\right) .
\end{aligned}
$$

It is elementary to know (cf. [TV]) that the action of the structure group $U(1) \times U(1)$ is irreducible on each of these factors

$$
\mathcal{L}\left(\pi_{1}-\pi_{2}\left(J_{\tau}\right)\right), \mathcal{W}_{7}\left(J_{\tau}^{\prime}\right), \mathcal{W}_{9}\left(J_{\tau}^{\prime}\right), \mathcal{W}_{A}, \mathcal{W}_{B}, \mathcal{W}_{C}, \mathcal{W}_{D}
$$

Q.E.D. 
We end this paper with some remarks.

Remarks. (A) Let $M$ be a 4-manifold which carries a field $\tau$ of 2-planes and a riemannian metric $g_{o}$. Associated with $\tau$, choose a local orthonormal frame $\left\{e_{1}, e_{2}, e_{3}, e_{4}\right\}$ at each point $x$ of $M$ with respect to $g_{o}$, which satisfies (4-3): $e_{1}, e_{2} \in \tau_{x}, e_{3}, e_{4} \in \nu_{x}$. In terms of the riemannian metric $g_{o}$ and the globally defined field $\tau$, we can construct a pseudo-riemannian metric $h$ of signature $(++--)$ as follows: for $X$, $Y \in \mathfrak{X}(M)$

$$
\begin{aligned}
h_{o}(X, Y)=g_{o}(X, Y) & -2 g_{o}\left(X, e_{3}\right) \cdot g_{o}\left(X, e_{3}\right) \\
& -2 g_{o}\left(X, e_{4}\right) \cdot g_{o}\left(X, e_{4}\right) .
\end{aligned}
$$

Such a metric $h_{o}$ does not depent on a particular frame with property (4-3), but only on the field $\tau$ of 2-planes, i.e., invariant by (4-6). In the above formula, if we take the $\left(J_{\tau}, J_{\tau}^{\prime}\right)$-invariant metric $g$ instead of $g_{o}$, then the following metric

$$
\begin{aligned}
h(X, Y)=g(X, Y) & -2 g\left(X, e_{3}\right) \cdot g\left(X, e_{3}\right) \\
& -2 g\left(X, e_{4}\right) \cdot g\left(X, e_{4}\right)
\end{aligned}
$$

is also $\left(J_{\tau}, J_{\tau}^{\prime}\right)$-invariant, and therefore we obtain a quadruple $(M, h$, $\left.J_{\tau}, J_{\tau}^{\prime}\right)$, a pseudo-riemannian version of the quadruple $\left(M, g, J_{\tau}, J_{\tau}^{\prime}\right)$. For such a pseudo-riemannian 4-manifold $\left(M, h, J_{\tau}, J_{\tau}^{\prime}\right)$ with the structure group $G=U(1) \times U(1)$, the bundle of pseudo-curvature tensors also splits into eleven irreducible factors in a similar way to that of the riemannian case in Theorem 7-2.

(B) It is highly expected that there may exist some intimate relations among the integrability conditions of fields of 2-planes (giving rise to 2dimensional foliations), the integrability of two kinds of almost complex structures (giving rise to two kinds of complex structures with opposite orientations), and the parallelizability of the two kinds of almost complex structures on 4-manifolds with fields of 2-planes. We shall discuss this issue elsewhere.

\section{References}

[A] M. F. Atiyah, Vector fields on manifolds, Arbeitsgemeinshaft für Forshung des Landes Nordorhein-Westfalen, Heft 200 (1970), Westdeutscher Verlag, Köln, Opladen.

[AHS] M. F. Atiyah, N. Hitchin and I.M. Singer, Self-duality in four-dimen -sional Riemannian geometry, Proc. R. Soc. London, A 308 (1982), 425-461. 
[BPV] W. Barth, C. Peters and A. Van de Ven, "Compact complex surfaces", Springer Verlag, Berlin Heidelberg New York Tokyo, 1984.

[DK] S. K. Donaldson and P. B. Kronheimer, "The Geometry of FourManifolds", Oxford University Press, Oxford, 1990.

$[\mathrm{HH}] \quad$ F. Hirzebruch and H. Hopf, Felder von Flächenelementen in 4-dimensionalen Mannigfaltigkeiten, Math. Ann., 136 (1958), 156-172.

[M1] Y. Matsushita, Fields of 2-planes on simply-connected 4-manifolds, Math. Ann., 280 (1988), 687-689.

[M2] Fields of 2-planes and two kinds of almost complex structures on compact 4-dimensional manifolds, Math. Z., 207 (1991), 281-291.

[S] N. Steenrod, "Topology of Fibre Bundles", Princeton University Press, Princeton, 1951.

[TV] F. Tricceri and L. Vanhecke, Curvature tensors on almost Hermitian manifolds, Trans. Amer. Math. Soc., 267 (1981), 365-398.

[W] W.-T. Wu, Sur les classes caractéristiques des structures fibrées sphériques, Actual. Sci. industr., 1183 (1952).

Department of Applied Mathematics and Physics

Faculty of Engineering

Kyoto University

Kyoto 606-01

Japan 